\title{
MICROENCAPSULATION OF GRAPE SEED OIL (VITIS VINIFERA L.) WITH GUM ARABIC AS A COATING POLYMER BY CROSSLINKING EMULSIFICATION METHOD
}

\author{
SILVIA SURINI, FARIHA ULFAH AZZAHRAH, DELLY RAMADON
}

Laboratory of Pharmaceutics and Pharmaceutical Technology Development, Faculty of Pharmacy, Universitas Indonesia, Depok, West Java, 16424, Indonesia

Email: silvia@farmasi.ui.ac.Id

Received: 03 Dec 2017, Revised and Accepted: 21 Sep 2018

\section{ABSTRACT}

Objective: Grape seed oil (GSO) from Vitis vinifera L. is a liquid vegetable oil which has been used mainly for its linoleic acid content. However, there are many efforts to convert the liquid form of the oil into a solid form due to the instability under storage condition. The aim of this study was to convert GSO into the solid microcapsules by emulsion crosslinking method with gum arabic as a coating polymer.

Methods: The GSO was formulated with gum arabic in the ratios of 1:2, 1:3, 1:4, and 1:5. Gum arabic solution was emulsified with GSO using Span 80 and glutaraldehyde. The emulsion was dropped into a beaker glass of isopropyl alcohol to form microcapsules. The microcapsules were dried at $70^{\circ} \mathrm{C}$. Then, they were characterized in terms of morphology, particle size, swelling index, water content, and entrapment efficiency.

Results: The produced microcapsules of GSO showed white yellowish color and spherical shape. The particle size of F1, F2, F3 and F4 microcapsules were $69 \mu \mathrm{m}, 82 \mu \mathrm{m}, 125 \mu \mathrm{m}$, and $131 \mu \mathrm{m}$, respectively. The water content of the F1-F4 ranged from $4.37 \pm 0.34$ to $5.70 \pm 0.92 \%$ and swelling indexes were ranged from $5.54 \pm 0.01$ to $5.94 \pm 0.04$. The value of entrapment efficiency of F1, F2, F3, and F4 were $17.33 \pm 0.603,20.73 \pm 0.678$, $34.22 \pm 1.195$, and $67.15 \pm 2.019 \%$, respectively.

Conclusion: The results of this investigation showed that GSO could be converted into the solid spherical microcapsules by emulsion crosslinking method using gum arabic. Taken together, this study has provided the most promising formulation of GSO microcapsules for further production in pharmaceutical industry.

Keywords: Crosslinking emulsification, Grape seed oil, Gum arabic, Microcapsules, Microencapsulation

(C) 2018 The Authors. Published by Innovare Academic Sciences Pvt Ltd. This is an open access article under the CC BY license (http://creativecommons.org/licenses/by/4.0/) DOI: http://dx.doi.org/10.22159/ijap.2018v10i6.24093

\section{INTRODUCTION}

Grapevine (Vitis vinifera L.) is one of the largest fruit crops in the world with a huge production in 2006 [1]. This fruit contains many beneficial compounds for body health, such as flavonoids, catechin and poly unsaturated fatty acids (PUFA) [2]. Most people use their flesh and throw the seeds as residues. However, the seeds themselves have a high value in commodities because of their advantages [3].

Grape seed oil (GSO) is a vegetable oil with a high content of antioxidant substances. This oil provides many benefits to the body because of its content, linoleic acid. Other useful compounds in GSO are tannins, phytosterols, flavonoids and carotenoids [4]. Moreover, GSO has been widely used in food products offering a good flavour [5], but it has a stability problem. GSO is unstable at room temperature (22 ${ }^{\circ} \mathrm{C}$ ), and when it is exposed by light [6]. Thus, the development of GSO formulation is important to increase its thermal stability.

One of the strategies is by formulating GSO into microcapsules. Microencapsulation is a method used to coat or encapsulate solid, gaseous or liquid active ingredients with one or more polymers [7]. This method has been extensively used in pharmaceutical industries to control the release properties or the availability of coated materials [8]. Moreover, microencapsulation can be utilized to convert the liquid into a solid form, such as GSO [9], so this technique could be applied to increase the thermal stability of GSO.

There are several methods for manufacturing microcapsules. One of the method is crosslinking emulsification. In this method, GSO would be emulsified with a hydrophilic polymer, and then crosslinked with a crosslinker. Gum arabic is a polymer that has a function as a coating material in the manufacture of GSO microcapsules. This gum is non-toxic, safe for the body, and able to be used as an emulsifier, so it might improve the stability of the emulsion. Considering the fact that gum arabic has a low viscosity, gum arabic is unable to perfectly coat GSO if it is used solely [10]. In the earlier studies, Patel and Patya (2013) reported that a crosslinking of gum arabic with glutaraldehyde $50 \%$ was required to increase the viscosity as well as to lower the hydrophilicity of gum arabic [9].

There were some researchers who worked on grape formulation [6, 9]. However, they used the flesh extract instead of the seeds [2]. In this study, GSO microcapsules were produced and then evaluated their morphology, yield, swelling index, water content, particle size distribution, levels of linoleic acid, chemical bonds by Fouriertransform infrared (FTIR) spectroscopy, and entrapment efficiency.

\section{MATERIALS AND METHODS}

\section{Materials}

GSO was purchased from Jian Hairui Natural Plant (China), linoleic acid was purchased from Sigma Aldrich (Singapura), gum arabic was purchased from Jumbo Trading (Thailand), glutaraldehyde $(50 \%$ v/v) was purchased from PT. Foton Prima Perkasa (Indonesia), Span 80, butylated hydroxy toluene (BHT), hydrochloric acid (HCl), sodium hydroxide $(\mathrm{NaOH})$, sodium chloride $(\mathrm{NaCl})$, isopropyl alcohol and aquadest was purchased from PT. Brataco (Indonesia).

\section{Preparation of microcapsules}

Formulations were prepared with different ratios of GSO and polymer. They were shown in table 1 . Crosslinked gum arabic microcapsules were prepared by emulsion crosslinking method with glutaraldehyde solution $(50 \% \mathrm{v} / \mathrm{v})$ as a crosslinking agent. An aqueous solution of gum arabic was prepared in aquadest. The aqueous phase was dispersed in GSO containing Span 80, an emulsifying agent, and butylated hydroxy toluene as an antioxidant. The mixture was stirred by using a homogenizer $\left(60^{\circ} \mathrm{C}\right)$ at $2000 \mathrm{rpm}$ for $30 \mathrm{~min}$. The $\mathrm{pH}$ of emulsion was adjusted to 3.7 with $\mathrm{HCl}$ solution $(7 \mathrm{~N}$ ). Glutaraldehyde solution was added to the emulsion. Then, the emulsion prepared was dropped into a beaker glass of isopropyl alcohol to form microcapsules. The microcapsules were then dried at $70^{\circ} \mathrm{C}$ and stored in desiccators before used and evaluated [9]. 
Table 1: Composition of GSO microcapsules

\begin{tabular}{|c|c|c|c|c|}
\hline Ingredients & F1 & F2 & F3 & F4 \\
\hline GSO (g) & 10 & 10 & 10 & 10 \\
\hline Gum arabic (g) & 20 & 30 & 40 & 50 \\
\hline Glutaraldehyde $50 \%(\mathrm{v} / \mathrm{v})(\mathrm{ml})$ & 5 & 7.5 & 10 & 12.5 \\
\hline
\end{tabular}

\section{Morphology of microcapsules}

Morphology of microcapsules was evaluated using a scanning electron microscope (SEM). Sample of GSO microcapsules were placed on the sample holder and coated with gold particle using the fine particle coater. Then, the morphology was visualized under the SEM at magnification of 20,000x [9].

\section{Percentage yield}

The yield was calculated as the percentage ratio between the weight of the microcapsules recovered from each batch and the total weight of drug and polymer used to prepare the same batch [9].

$$
\text { Percentage yield }=\frac{\text { Practical } \text { yield }}{\text { Theoretical } \text { yield }} \times 100
$$

\section{Swelling index}

The swelling index of microcapsules was determined by immersing the microcapsules obtained in a phosphate buffered saline (PBS) (pH 7.4) and allowed to swell for $24 \mathrm{~h}$. The weight of the swollen microcapsules was measured, and the swelling ratio was calculated according to the equation as follows:

$$
\text { Swelling index }=\frac{W-W 0}{W 0}
$$

Where $\mathrm{W}$ is the weight of the microcapsules after swelling in the medium, and WO is the initial weight of the microcapsule [9].

\section{Water content}

Water content of the microcapsules was determined by using a moisture analyzer. The apparatus was preheated for $10 \mathrm{~min}$ before used, then a sample of GSO microcapsules ( $1 \mathrm{~g}$ ) was placed on top of the aluminium container and measured at the temperature of $105^{\circ} \mathrm{C}$ [11].

\section{Particle size distribution}

The particle size distribution was measured using a particle size analyser (Mastersizer 3000 MAZ 6240) by laser diffraction technique. Samples were dispersed in isopropyl alcohol under constant stirring. The average diameter and the equivalent volume diameters at 10,50 and $90 \%$ cumulative volume were determined [12].

\section{Crosslinking confirmation}

Fourier transform infrared spectroscopy (FTIR) spectra was obtained using an FTIR spectrometer (Shimadzu® FTIR-8400). This analysis was conducted to identify the chemical bonds formed between gum arabic and glutaraldehyde. The sample was prepared by potassium bromide (KBr) disk method and scanned over the range of $400-4000 \mathrm{~cm}^{-1}$. The spectra were recorded to identify certain characteristic bonds in the compounds that indicated the formation of new compounds [9].

\section{Entrapment efficiency}

A total of $2 \mathrm{~g}$ GSO microcapsules was placed into a beaker glass. A volume of $10 \mathrm{ml} \mathrm{HCl}$ was added into the beaker glass. The solution was extracted using diethyl ether and petroleum benzene, and this step was repeated three times. The supernatant was placed into a glass and reheated on a water bath until all of the water evaporated and oil residue was remained [13]. Entrapment efficiency of GSO microcapsules was measured by Mojonnier Tester and calculated according to the equation as follows:

$$
\text { Entrapment efficiency }=\frac{\text { Grape seed oil extract }}{\text { Theoretical grape seed oil }} \times 100 \%
$$

\section{Determination of linoleic acid}

A total of $20 \mathrm{mg}$ GSO extract was placed into a sample tube. A volume of $1 \mathrm{ml} \mathrm{NaOH} 0.5 \mathrm{~N}$ in methanol was added into the tube and then flowed with nitrogen. The tube was heated in a water bath for $20 \mathrm{~min}$. A volume of $2 \mathrm{ml}$ boron trifluoride was placed into the tube and reheated for $20 \mathrm{~min}$. The tube was then cooled, added with $2 \mathrm{ml}$ of saturated $\mathrm{NaCl}$ and $1 \mathrm{ml}$ of hexane, then homogenized using a vortex. The mixed solution was allowed to stand for $15 \mathrm{~min}$. A $1.0 \mu \mathrm{L}$ n-hexane layer containing methyl linoleate was drawn and injected into a gas chromatographic device [14]. The optimized gas chromatographic system was shown in table 2.

\section{Table 2: Gas chromatographic condition}

\begin{tabular}{ll}
\hline Parameter & Result \\
\hline Column & Cyanopropil methyl sil (capilary column) \\
Mobil phase & Nitrogen \\
Detector & Flame Ionization Detector \\
Injector temperature & $220^{\circ} \mathrm{C}$ \\
Detector temperature & $240^{\circ} \mathrm{C}$ \\
\hline
\end{tabular}

Note: The initial column temperature was $125{ }^{\circ} \mathrm{C}$, and an increase rate of $10{ }^{\circ} \mathrm{C} / \mathrm{min}$ up to $185{ }^{\circ} \mathrm{C}$ was employed. Then, the temperature was increased again by $5^{\circ} \mathrm{C} / \mathrm{min}$ until $205^{\circ} \mathrm{C}$, and the last increase was $3{ }^{\circ} \mathrm{C} / \mathrm{min}$ to $225^{\circ} \mathrm{C}$. The flow rate of the mobile phase was set at $30.0 \mathrm{ml} / \mathrm{min}$.

\section{RESULTS AND DISCUSSION}

\section{Formulation and characterization of GSO microcapsules}

The characteristics of the GSO microcapsules were listed in table 3. GSO microcapsules were prepared by emulsion crosslinking method, which was dropping the GSO emulsion into isopropyl alcohol. This method is relatively easy to implement and capable to produce relatively stable globules during the storage. There are some variables that may influence the production of GSO microcapsules, such as amount of polymer (gum arabic) and crosslinking agent.

Table 3: Characteristic of GSO microcapsules

\begin{tabular}{lllll}
\hline Sample & Water content (\%) & Percentage yield (\%) & Particle size average* $(\boldsymbol{\mu m})$ & Swelling index (\%) \\
\hline F1 & $4.37 \pm 0.34$ & $60.33 \pm 0.34$ & 69 & $553.70 \pm 0.61$ \\
F2 & $4.68 \pm 0.46$ & $61.08 \pm 0.29$ & 82 & $568.97 \pm 1.25$ \\
F3 & $4.98 \pm 0.17$ & $78.07 \pm 0.11$ & 125 & $588.79 \pm 1.73$ \\
F4 & $5.70 \pm 0.92$ & $83.89 \pm 0.04$ & 131 & $594.51 \pm 3.49$ \\
\hline
\end{tabular}

Each value represents the mean \pm standard deviation of three determinations, *Particle size average was in one batch production of each microcapsules. 


\section{Shape and morphology of microcapsules}

The morphology of GSO indicated that all prepared microcapsules had a spherical shape without any pores on the surface as shown in fig. 1. The surface morphology performed for all formulations were in a magnification of $20000 x$. The shape of microcapsules can be affected by the temperature and the time of drying [15]. It showed that all formulations exhibited no porous formation, thus the gum arabic as a coating material was capable in protecting the active substance from environmental influences which might reduce the stability, such as light and air [15-16].

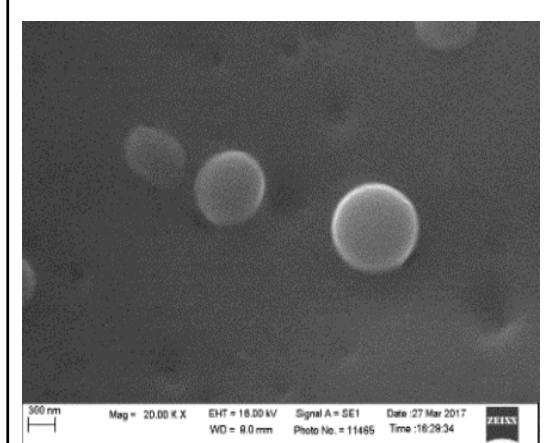

(a)

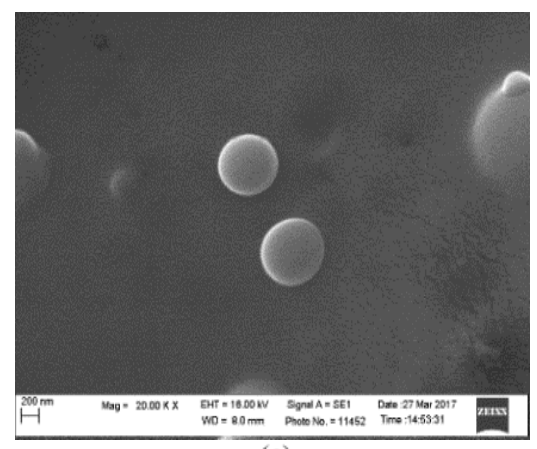

(c)



(b)

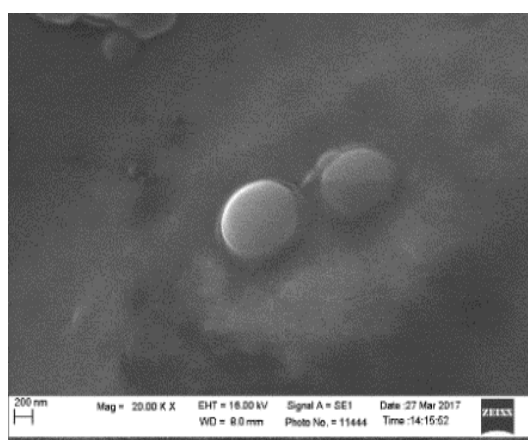

(d)

Fig. 1: Scanning electron micrographs of GSO microcapsule (a) F1, (b) F2, (c) F3 and (d) F4, with a magnification of $20000 x$

\section{Percentage yield}

The percentage yield obtained in various batches was between 60 $84 \%$, with the highest yield value was from F4. It was observed that as the polymer ratio in the formulation increased, the product yield increased [19]. The low percentage yield may be due to the microcapsules lost during the washing process.

\section{Swelling index}

Table 3 showed the swelling index of the GSO microcapsules in PBS. The swelling index was ranged from 5.53 to 5.94. The swelling of the microcapsules occurred due to the hydration of gum arabic in an aqueous environment. The higher polymer concentration in formulation would give higher value of water content and swelling index. The value of the microcapsules swelling index showed that gum arabic used as the coating agent had the characteristic of high power expander because it is a hygroscopic polymer $[17,18]$.

\section{Moisture content}

The measurement of the moisture content of GSO microcapsules was given in table 3. It shows that $\mathrm{F} 4$ had the highest moisture content with the value of $5.7 \%$. It might be caused by the higher contain of gum arabic in the formulation, which was hydrophilic polymer, thus it was easier for F4 to contact with moist.

\section{Particle size distribution}

Fig. 2 showed the particle size distribution of the GSO microcapsules, and it was ranged between $2-500 \mu \mathrm{m}$. particularly, the particle size of $\mathrm{F} 1$ and $\mathrm{F} 2$ was $51.8 \mu \mathrm{m}(5.68 \%)$ and $66.9 \mu \mathrm{m}(6.44 \%)$, respectively. On the other hand, the particle size distribution of $\mathrm{F} 3$ and F4 were in the range $\geq 0998-111 \mu \mathrm{m}$ with a percentage of
6.21\%. The microcapsules particle size ranged between $69-131 \mu \mathrm{m}$ as shown in table 4.

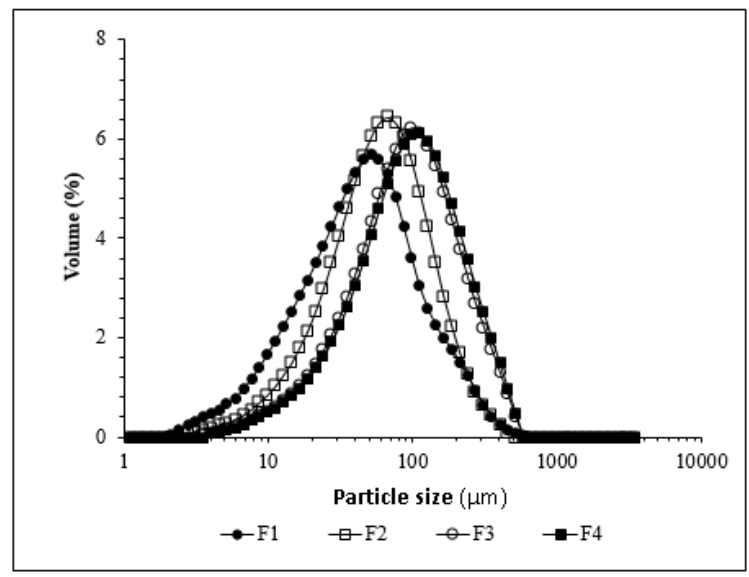

Fig. 2: Particle size distribution of GSO microcapsules

One of the important parameters in microcapsules preparation is particle size. Parameter used for the particle size distribution measurement were dv or diameter based on volume, because it can describe heterogeneity of particle size of the sample [20]. The results of the particle size distribution for all formulations, from the smallest to largest particle size, was $\mathrm{F} 1<\mathrm{F} 2<\mathrm{F} 3<\mathrm{F} 4$ (table 4 ). It 
revealed that $\mathrm{F} 1$ had the smallest dv90 at $155 \mu \mathrm{m}$. It suggested that the concentration of GSO in the microcapsules affected the particle size. The higher concentration of GSO might cause the larger microcapsules particle size [21]. The particle size could also be influenced by the speed of stirring and the drug concentration in microcapsule [22-23]. Furthermore, the particle size distribution might be affected by the dispersion medium and dispersed phase due to the viscosity increase of the polymer. If the polymer concentration increases, the relative viscosity would rise, and it might affect and increase the mean particle size [24]. Dynamic light scattering (DLS) can be used to determine particle size which followed by the value of uniformity. The higher the stirring speed, the smaller the size of the microcapsules formed [22-25].

Table 4: The of particle size measurement of the GSO microcapsules*

\begin{tabular}{lllll}
\hline Formula & $\mathbf{d v}_{\mathbf{1 0}}(\boldsymbol{\mu m})$ & $\mathbf{d v}_{\mathbf{5 0}}(\boldsymbol{\mu m})$ & $\mathbf{d v}_{\mathbf{9 0}}(\boldsymbol{\mu m})$ & $\mathbf{D}_{\text {mean }}$ volume $(\boldsymbol{\mu m})$ \\
\hline 1 & 12.1 & 47.5 & 155 & 69 \\
2 & 19.3 & 63.6 & 167 & 82 \\
3 & 27.5 & 96.4 & 264 & 125 \\
4 & 28.7 & 102.0 & 279 & 131 \\
\hline
\end{tabular}

*The data was from measurements using Mastersizer 3000 and each formulation was in one batch production of each microcapsules.

\section{Crosslinking confirmation}

The chemical interaction in the cross-linked gum arabic were observed by FTIR spectroscopy. The infrared spectra was shown in fig. 3. In the cross-linked gum arabic spectra, there was an absorption band in the region of $3200-3600 \mathrm{~cm}^{-1}$ which indicated the presence of $\mathrm{OH}$-groups in hydrogen bonds. Based on the fig. 3 , it can be seen that the hydrogen bonding intensity of the cross-linked gum arab was lower than natural gum. It was due to the fact that the OH-groups on gum arabic undergone a substitution by other groups. The spectrum also showed the characteristic bands of $\mathrm{C}-\mathrm{O}$ around $1148 \mathrm{~cm}^{-1}$. Other absorptions also appeared at the wavenumber of $1738 \mathrm{~cm}^{-1}$.
In terms of chemical bonds formed, the absorptions appeared at the wavenumber of $1738 \mathrm{~cm}^{-1}$. The absorption band at $1738 \mathrm{~cm}^{-1}$ expressed the carbonyl group which could be formed due to the formation of a new acetal groups [26]. According to Distantina and Fahrurrozi (2013), the emergence of new absorption is due to the formation of the acetal group due to the interaction between the aldehyde groups on the glutaraldehyde with hydrogen in gum arabic [27]. Patel (2013) also stated that crosslinked gum arabic would result in a new cluster due to the interaction between gum arabic and glutaraldehyde in the infrared spectrum. Based on the results obtained, it showed that there had been a reaction between gum arabic with glutaraldehyde [9].



Fig. 3: FTIR-spectrum (a) crosslinked gum arabic and (b) a natural gum arabic

\section{Entrapment efficiency}

Table 5 showed the entrapment efficiency of the GSO microcapsules, which was ranged between 17.33 to $67.15 \%$. F1, which was composed with the lowest polymers concentration, showed the lowest entrapment efficiency value of $17.33 \%$. On the other hand, F4 with the highest polymer concentration, had the highest entrapment efficiency of $67.15 \%$.

In this study, the entrapment efficiency result was similar to Ramadon (2017) [21], where the entrapment efficiency value would increase in accordance with the increase of polymer concentration. The high polymer concentration would hinder homogeneous distribution of the glutaraldehyde leading to the formation of larger microcapsules with reduced drug content and entrapment efficiency [24]. Increasing the polymer concentration would result in an increase of viscosity of the solution and the precipitation of the polymer, then it accelerates the dispersed phase to prevent the drug diffuses out $[28,30]$. F1 had a low entrapment efficiency because the GSO was degraded during the manufacturing and analytical process. However, the concentration of free GSO were not calculated, so there is no supporting data to prove it. 
Table 5: The entrapment efficiency and the content of linoleic acid of the grape seed oil microcapsules

\begin{tabular}{lll}
\hline Sample & Entrapment Efficiency (\%) & Content of linoleci acid (\%) \\
\hline F1 & $17.33 \pm 0.60$ & $32.38 \pm 1.68$ \\
F2 & $20.73 \pm 0.68$ & $32.85 \pm 0.82$ \\
F3 & $34.22 \pm 1.20$ & $33.21 \pm 0.94$ \\
F4 & $67.15 \pm 2.02$ & $34.75 \pm 0.58$ \\
\hline
\end{tabular}

Each value represents the mean \pm standard deviation of three determinations.

\section{Determination of linoleic acid}

The content of linoleic acid in the GSO and GSO-loaded microcapsules prepared was displayed in table 5. The pure GSO contained linoleic acid as amount of $50.28 \%$. The content of linoleic acid in each formula of the GSO microcapsules was decreased around $17 \%$. This result might be affected by the destruction of the linoleic acid in GSO during the microcapsules preparation process by heating, and as a result of oxidation of linoleic acid in GSO by crosslinking agent which was involved in formulation process [11].

\section{CONCLUSION}

The grape seed oil (GSO) was successfully encapsulated by crosslinking emulsification method with gum arabic as the coating agent. Thus, these findings have significant implications for the understanding of how the crosslinking emulsification method could be a good approach to encapsulate liquid material for producing a solid powder form.

\section{ACKNOWLEDGEMENT}

The authors gratefully acknowledge to Directorate of Research and Community Engagements of Universitas Indonesia for PITTA research grant.

\section{AUTHORS CONTRIBUTION}

All the authors have contributed equally

\section{CONFLICTS OF INTERESTS}

The authors have no confict of interest

\section{REFERENCES}

1. Maier T, Schieber A, Kammerer DR, Carle R. Residues of grape (Vitis vinifera L.) seed oil production as a valuable source of phenolic antioxidants. Food Chem 2009;112:551-9.

2. Iannone M, Mare R, Paolino D, Gagliardi A, Froiio F, Cosco D, et al. Characterization and in vitro anticancer properties of chitosan-microencapsulated flavan-3-ols-rich grape seed extracts. Int J Biol Macromol 2017;104:1039-45.

3. Passos CP, Silva RM, Da FA, Coimbra MA, Silva CM. Supercritical fluid extraction of grape seed (Vitis vinifera L.) oil effect of the operating conditions upon oil composition and antioxidant capacity. Chem Eng J 2010;160:634-40.

4. Garavaglia J, Markoski MM, Oliveira A, Marcadenti A. Grape seed oil compounds: biological and chemical actions for health. Nutr Metab Insights 2016;9:59-64.

5. Bail S, Stuebiger G, Krist S, Unterweger H, Buchbauer G. Characterisation of various grape seed oils by volatile compounds, triacylglycerol composition, total phenols and antioxidant capacity. Food Chem 2008;108:1122-32.

6. Lachman J, Hejtmankova A, Kotikova Z, Dedina M, Stralkova R, Hönig V. Stability of grape seed oil and its antioxidant tocotrienols. Adv Materials Res 2014;1032:370-3.

7. Dubey R, Shami TC, Bhasker Rao KU. Microencapsulation technology and applications. Def Sci J 2009;59:82-95.

8. Venkatesan P, Manavalan R, Valliappan K. Microencapsulation: a vital technique in novel drug delivery system. J Pharm Sci Res 2009;1:26-35.

9. Patel P, SS P. Preparation and characterization of crosslinked gum acacia microspheres by single step emulsion in-situ polymer crosslinking method: apotential vehicle for controlled drug delivery. Res Rev J Pharm Pharm Sci 2013;2:40-8.

10. Khalil Azzaoui, Premier M, Hammouti B, Universit AL, Premier $M$. The gum arabic in the southern region of morocco. J Chem 2015;3:99-107.

11. Surini S, Nursatyani K, Ramadon D. Gel formulation containing microcapsules of grape seed oil (Vitis vinifera L.) for skin moisturizer. J Young Pharm 2018;10:41-7.

12. Pandta HX, Nguyen CN. Formulation and evaluation of microcapsules of trihexyphenidyle hydrochloride by solvent evaporation method. Int J Pharm Pharm Sci 2012;3:539-44.

13. Association of Official Analytical Chemist. Official Method of Analysis International. AOAC International; 2006.

14. Damayanti S, Sigi VA, Julianti E. Transesterification of linoleic acid in grape seed (Vitis vinifera $\mathrm{L}$.) oiland its analytical method development using gas chromatography. Int J Pharm Pharm Sci 2014;6:4-7.

15. Freitas S, Merkle H, Gander B. Microencapsulation by solvent extraction/evaporation: reviewing the state of the art of microsphere preparation process technology. J Controlled Release 2005;102:313-32.

16. Goswami S, Naik S. Natural gums and its pharmaceutical application. J Sci Innovative Res 2014;3:112-21.

17. Verbeek C. Products and applications of biopolymers. Croatia: InTech; 2012. p. 9-12.

18. Fernandes R, Borges S, Botrel D. Gum Arabic/Starch/Maltodextrin/Inulin as wall materials on the microencapsulation of rosemary essential oil. Carbohydr Polym 2014;101:524-32.

19. Vidal JL, Avello LM, Loyola CC, Campos PJ, Aqueveque MP, R Dungan S, et al. Microencapsulation of maqui (Aristotelia chilensis Molina Stuntz) leaf extracts to preserve and control antioxidant properties. Chil J Agric Res 2013;73:17-23.

20. Malvern. A basic guide to particle characterization. United Kingdom: Malvern; 2012.

21. Ramadon D, Wirarti GA, Anwar E. Novel transdermal ethosomal gel containing green tea (Cammelia sinensis L. Kuntze) leaves extract: formulation and in vitro penetration study. J Young Pharm 2017;9:336-40.

22. O'Donnell P, McGinity J. Preparation of microspheres by the solvent evaporation technique. Adv Drug Delivery Rev 1997;28:25-42.

23. Anwar E, Utami T, Ramadon D. Transfersomal gel containing green tea (Camellia sinensis L. Kuntze) leaves extract: increasing in vitro penetration. Asian J Pharm Clin Res 2017;10:294.

24. Pavanveena C, Kavitha K, Anil KSN. Formulation and evaluation of trimetazidine hydrochloride loaded chitosan microsphers. Int J Appl Pharm 2010;2:11-4.

25. Kuck L, Norena C. Microencapsulation of grape (Vitis labrusca var. Bordo) skin phenolic extract using gum arabic, polydextrose, and partially hydrolyzed guar gum as encapsulating agents. Food Chem 2016;194:569-76.

26. Ramachandran S, Nandhakumar S, Dhanaraju M. Formulation and characterization of glutaraldehyde cross-linked chitosan biodegradable microspheres loaded with famotidine. Tropical J Pharm Res 2011;10:309-16.

27. Distantina S, Rochmadi R, Fahrurrozi M, Wiratni W. Preparation and characterization of glutaraldehydecrosslinked kappa carrageenan hydrogel. Eng J 2013;17:57-66. 